\title{
Evolution of Metastable Structures at Bimetallic Surfaces from Microscopy and Machine-Learning Molecular Dynamics
}

Jin Soo Lim, ${ }^{\dagger}$ Jonathan Vandermause, ${ }^{\ddagger}$ Matthijs A. van Spronsen, ${ }^{\dagger}, \S$ Albert Musaelian, "Yu Xie, "Lixin Sun, "Christopher R. O'Connor, ${ }^{\dagger}$ Tobias Egle, ${ }^{\dagger} \rrbracket$ Nicola Molinari, Jacob Florian, ${ }^{\dagger}$ Kaining Duanmu,, Robert J. Madix, $₫$ Sautet, $\|, \perp$ Cynthia M. Friend, ${ }^{\dagger}, \boldsymbol{\Phi}$ and Boris Kozinsky $*, \mathbf{\Phi}, \#$

$\dagger$ Department of Chemistry and Chemical Biology, Harvard University, Cambridge, MA 02138, USA

$\ddagger$ Department of Physics, Harvard University, Cambridge, MA 02138, USA

IJohn A. Paulson School of Engineering and Applied Sciences, Harvard University, Cambridge, MA 02138, USA

$\S$ Materials Sciences Division, Lawrence Berkeley National Laboratory, Berkeley, CA 94720, USA

||Department of Chemical and Biomolecular Engineering, University of California, Los Angeles, Los Angeles, California 90095, USA

$\perp$ Department of Chemistry and Biochemistry, University of California, Los Angeles, Los Angeles, California 90095, USA

\#Robert Bosch LLC, Research and Technology Center, Cambridge, MA 02142, USA

E-mail: bkoz@seas.harvard.edu 


\begin{abstract}
Restructuring of interfaces plays a crucial role in materials science and heterogeneous catalysis. Bimetallic systems, in particular, often adopt very different composition and morphology at surfaces compared to the bulk. For the first time, we reveal a detailed atomistic picture of long-timescale restructuring of $\mathrm{Pd}$ deposited on $\mathrm{Ag}$, using microscopy, spectroscopy, and novel simulation methods. By developing and performing accelerated machine-learning molecular dynamics followed by an automated analysis method, we discover and characterize previously unidentified surface restructuring mechanisms in an unbiased fashion, including $\mathrm{Pd}-\mathrm{Ag}$ place exchange and $\mathrm{Ag}$ pop-out, as well as step ascent and descent. Remarkably, layer-by-layer dissolution of $\mathrm{Pd}$ into $\mathrm{Ag}$ is always preceded by an encapsulation of $\mathrm{Pd}$ islands by $\mathrm{Ag}$, resulting in a significant migration of $\mathrm{Ag}$ out of the surface and a formation of extensive vacancy pits within a period of microseconds. These metastable structures are of vital catalytic importance, as Ag-encapsulated Pd remains much more accessible to reactants than bulk-dissolved Pd. Our approach is broadly applicable to complex multimetallic systems and enables the previously intractable mechanistic investigation of restructuring dynamics at atomic resolution.
\end{abstract}

\title{
Introduction
}

Dynamic restructuring of complex interfaces is ubiquitous in multicomponent materials and can have a significant impact on their physical and chemical properties. In particular, elemental segregation at grain boundaries, dislocations, and surfaces can dramatically alter a range of functional properties, such as fracture strength, plastic deformation, corrosion resistance, and catalytic activity. ${ }^{1-3}$ In heterogeneous catalysis, bimetallic and dilute alloy catalysts have garnered much attention due to their potential in enhancing both the activity and the selectivity of hydrogenation reactions. ${ }^{4}$ In dilute $\mathrm{Pd} / \mathrm{Ag}$, for example, $\mathrm{Pd}$ initiates the catalytic cycle as the active site for $\mathrm{H}_{2}$ dissociation, and the selective hydrogenation 
ensues on a more inert Ag host. ${ }^{5}$ These alloy surfaces are inherently dynamic at reaction temperatures of interest: compared to the original synthesized state, the surface composition and morphology can differ dramatically after annealing or during the reaction. ${ }^{6-10}$ Such restructuring has recently been shown to play a central role in catalytic performance of several alloy systems, including $\mathrm{RhPd},{ }^{6} \mathrm{NiPt},{ }^{11} \mathrm{PdAg},{ }^{12}$ and $\mathrm{AgAu} .{ }^{13}$ Therefore, fundamental understanding of surface restructuring is crucial to enabling accurate prediction and engineering of catalyst performance. ${ }^{14}$

Mechanistic and kinetic studies of surface restructuring at the atomic level have remained scarce and challenging. ${ }^{15-21}$ Experimentally, it is very difficult to probe fast atomic motions with sufficient temporal resolution, even with techniques that possess surface and chemical sensitivities. Computationally, density functional theory (DFT) enables exploration of reaction pathways on model surfaces, ${ }^{22}$ but its high computational cost precludes its use for dynamical simulation of realistic systems beyond tens of picoseconds. Furthermore, exploration of large configurational space underlying restructuring processes quickly becomes intractable with brute force approaches. In this work, we overcome all of these limitations by combining microscopy, spectroscopy, and novel simulation methods (Fig. 1; see Experimental Section). Fast and large-scale machine-learning molecular dynamics (MD) is performed using our recently developed Gaussian process (GP) force field, ${ }^{23}$ spanning microseconds at first-principles accuracy. Furthermore, a new automated analysis method is used to discover and characterize key surface restructuring mechanisms (Fig. 1). Our streamlined approach enables an efficient and unbiased characterization of restructuring dynamics in complex multimetallic systems.

Surface restructuring of late-transition metals can be classified into three categories: (1) relaxation involving localized atomic displacements; ${ }^{30}$ (2) reconstruction involving intralayer atomic site changes; ${ }^{31}$ (3) segregation in alloys involving interlayer atomic site changes. ${ }^{32}$ All three processes aim to reduce the surface free energy arising from reduced surface atom coordination. In alloy systems, equilibrium distribution of surface and subsurface species 


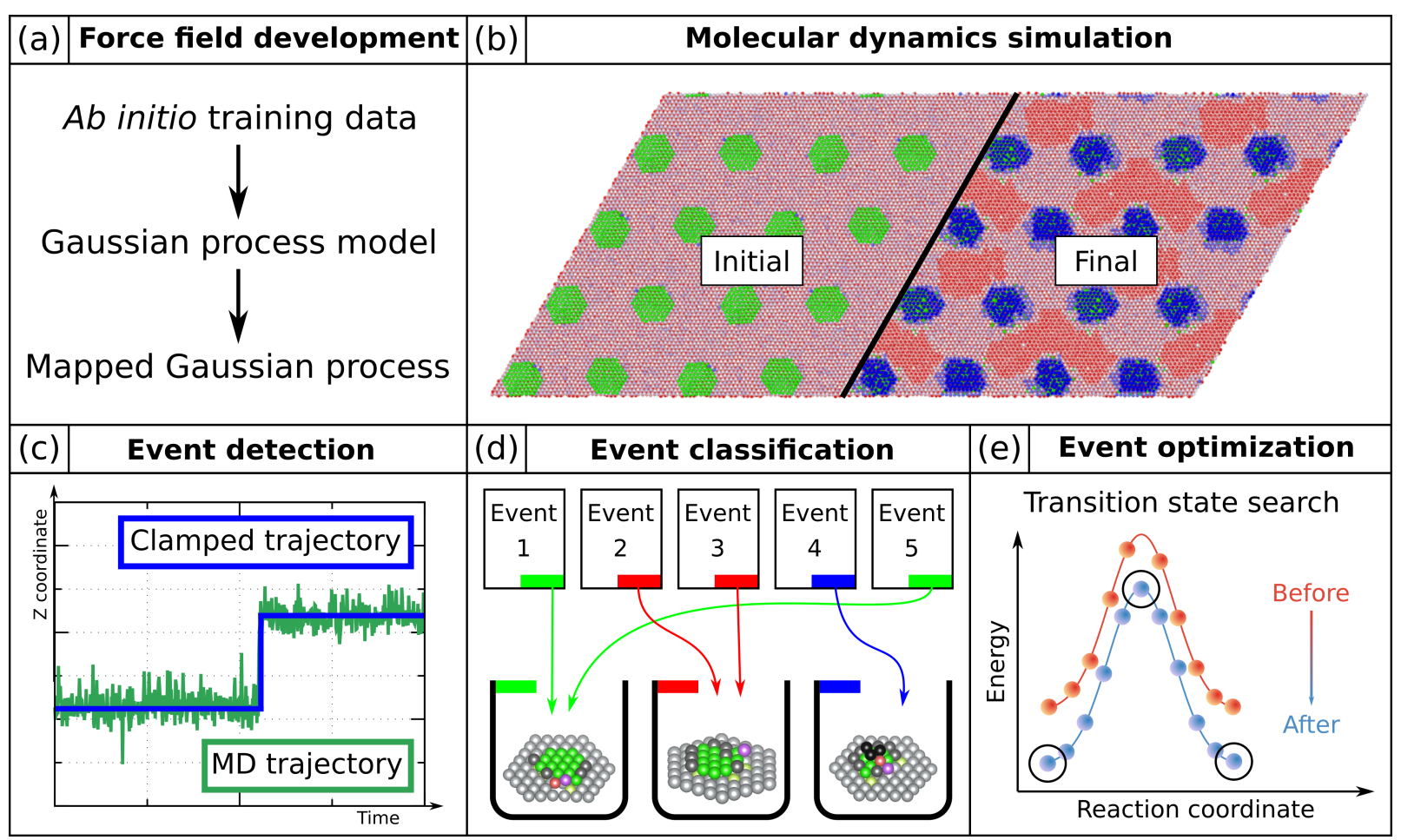

Figure 1: Automated characterization of surface restructuring events. (a) Gaussian process (GP) machine-learning force field is trained on DFT data. ${ }^{23}$ (b) The GP model is used to perform fast, large-scale, and long-timescale MD simulation of surfaces. (c) The raw MD trajectory is first "clamped" by assigning all atoms onto discrete FCC lattice sites. ${ }^{24-26}$ Then, an event is detected whenever the $z$-coordinate (normal to the surface) of any atom changes irreversibly over 1-2 ps. ${ }^{21}$ (d) The events are sorted into seven main classes ${ }^{21}$ based on changes in the atomic layer and the coordination number. (e) The event pathways are detailed using transition state modeling. ${ }^{27-29}$

can be modeled using the quasi-chemical approximation. ${ }^{33}$ The model assumes a chemical equilibrium according to select energetic contributions, which consist of the "three effects' rule" 32 (surface energy; strain energy; alloying energy) and the "chemical pump effect" 34 (adsorption energy of molecular species).

In bimetallic systems comprised of soft metals (e.g. Pt, Pd, Au, Ag), the major chemical complexity arises from elemental variations in the surface energy. In general, elements with fuller $d$-shells have lower surface energy and segregate to the surface under reducing conditions. For example, an inert metal of group $11(\mathrm{Cu}, \mathrm{Ag}, \mathrm{Au})$ has a full $d$-shell and segregates to the surface, while an active metal of group 10 (Ni, Pd, Pt) migrates to the subsurface, ultimately forming e.g. core@shell nanostructures. ${ }^{35}$ Based on prior studies using scanning 
tunneling microscopy (STM), X-ray photoelectron spectroscopy (XPS), and ab initio thermodynamics, $\mathrm{Pd}$ dissolves into the Ag bulk after mild heating beause of the higher surface energy of $\mathrm{Pd} .{ }^{10}$ However, the underlying mechanism and timescale have remained unclear.

To the best of our knowledge, the work herein provides the first direct atomistic observation and characterization of the long-timescale restructuring of a biemtallic surface. After annealing at a moderate temperature, monatomic $\mathrm{Pd}$ islands on $\mathrm{Ag}(111)$ evolve into a dramatically different state within a period of microseconds: a significant amount of $\mathrm{Ag}$ migrates out of the surface to encapsulate the $\mathrm{Pd}$ islands, resulting in extensive vacancy pits. We discover and characterize previously unidentified elementary restructuring events, including $\mathrm{Pd}-\mathrm{Ag}$ place exchange and $\mathrm{Ag}$ pop-out, as well as step ascent and descent. In contrast to the thermodynamically favored state where $\mathrm{Pd}$ is dispersed in the $\mathrm{Ag}$ bulk, these structures are metastable and prevail at mild temperatures. They are of vital catalytic importance, as Ag-encapsulated Pd remains much more accessible to reactants than bulkdissolved Pd. As such, our results provide an important foundation for engineering surface ensemble distributions toward optimal activity-selectivity balance. ${ }^{36}$

\section{Results and Discussion}

Four distinct stages have been established for the restructuring of $\mathrm{Pd}$ islands on $\mathrm{Ag}$ (Fig. 3): (1) Pd-Ag place exchange; (2) formation of PdAg alloy layer; (3) Ag pop-out and vacancy pit growth; (4) layer-by-layer Pd dissolution. Surprisingly, the first three stages produce nontrivial metastable structures that result from encapsulation of the $\mathrm{Pd}$ deposit by Ag and the growth of extensive vacancy pits, as observed in STM images (Fig. 2). As such, these stages comprise a kinetically distinct metastable dissolution mode, previously named "surfactant" mode in the literature ${ }^{37}$ due to the similarity to semiconductor growth. This mode precedes the direct layer-by-layer dissolution of Pd into the thermodynamically favored subsurface. Fig. 3 shows the evolution of a hexagonal $\mathrm{Pd}_{91}$ island on $\mathrm{Ag}(111)$ at 
As-deposited

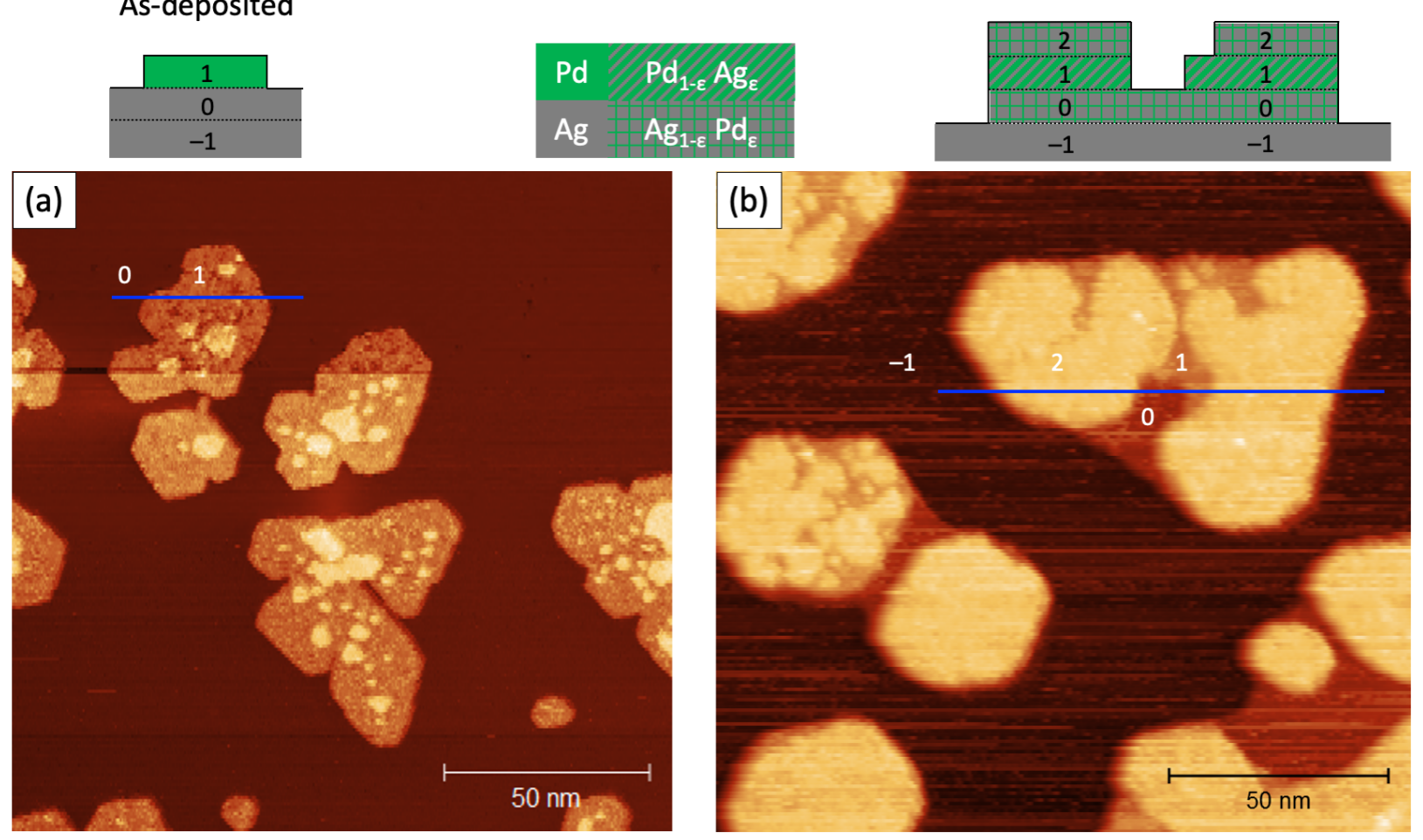

Figure 2: STM images demonstrate the restructuring of Pd islands deposited on $\mathrm{Ag}(111)$. Metastable structures form prior to complete dissolution of Pd into the Ag bulk. Schematic height profiles along the blue horizontal lines are depicted above the images, labeled by the layer heights: level -1 (subsurface); level 0 (surface); level 1 (deposit); level 2 (capping). Compositions derived from simulation (Fig. 3) are indicated by colored patterns for each layer (see top center legend), with $\epsilon \ll 1$ corresponding to the minority component. (a) Pd islands, deposited at $300 \mathrm{~K}$, consist mostly of monolayers at level 1. (b) After mild annealing at 400-450 K, the difference in the elemental surface energy results in encapsulation of the $\mathrm{Pd}$ islands by capping layers at level 2, along with extensive vacancy pits at level -1 .

$500 \mathrm{~K}$ over $2 \mu$ s of our simulation. In addition, Table 1 shows four main types of microscopic restructuring events discovered by our automated scheme (Sec. 2, SI). 

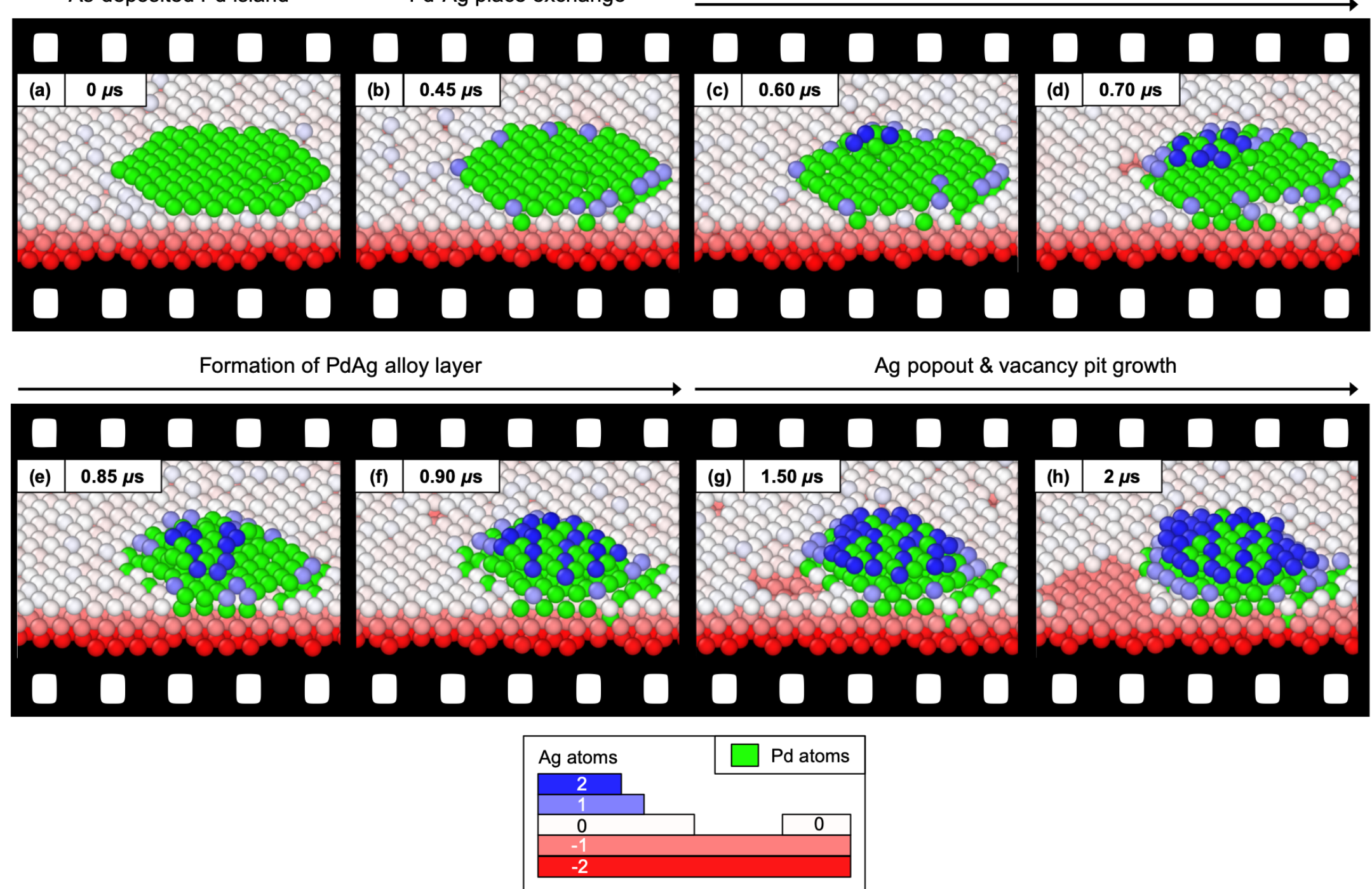

Figure 3: Frames of MD simulation showing the evolution of a $\mathrm{Pd}_{91}$ island on $\mathrm{Ag}(111)$ at $500 \mathrm{~K}$ over $2 \mu$ s. The system reaches a quasi-equilibrium after $2 \mu \mathrm{s}$. As depicted in the bottom schematic, Pd atoms are green, and Ag atoms are colored by the layer height, from red (level -2) to blue (level 2). The bottommost layer (level -3 ) is fixed in the simulation and hence omitted. (a) As-deposited hexagonal $\mathrm{Pd}_{91}$ island on $\mathrm{Ag}(111)$. (b) $\mathrm{Pd}$ undergoes a series of place exchange with the underlying Ag atoms around the island edges. (c-d) A few Ag atoms climb on top of the deposit and attempt to pull Pd atoms up to level 2. (e-f) $\mathrm{PdAg}$ alloy layer forms at level 2. (g-h) In the second half of the simulation, more Ag atoms migrate out of the surface to further encapsulate the cluster, resulting in a vacancy pit. See video here. 
Table 1: Four main classes of restructuring events underlying the restructuring of a Pd island on $\mathrm{Ag}(111)$ : (a) Pd-Ag direct exchange; (b) Ag pop-out; (c) Ag hopping ascent; (d) Pd exchange ascent. The structures (IS = initial state; TS = transition state; IMS = intermediate state; FS = final state) are fully optimized by the GP model, followed by DFT evaluation of the relative energies. Slab atoms are semi-transparent for clarity.

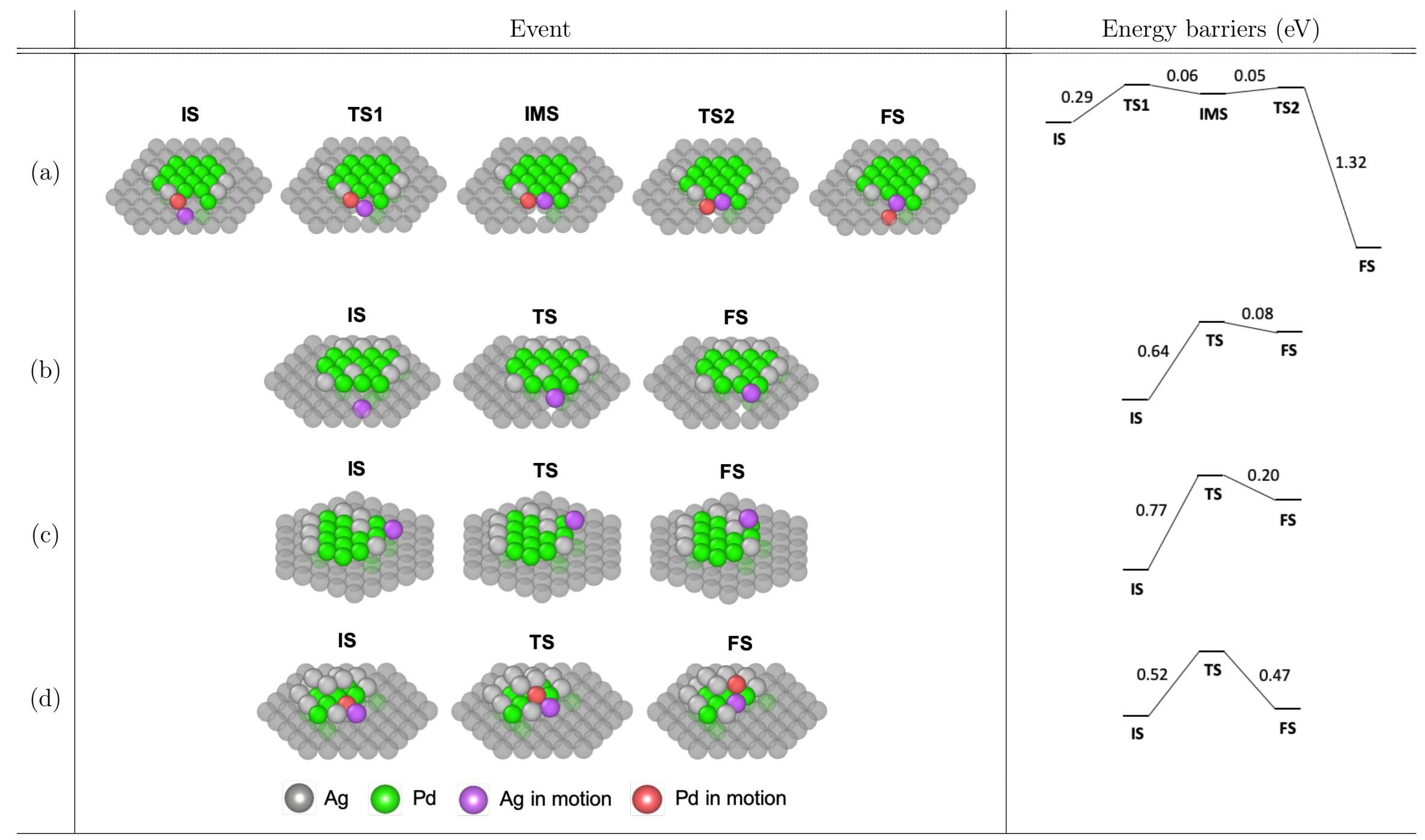


In the beginning, Pd undergoes a series of place exchange with the underlying Ag atoms around the island edges [Fig. 3(b)]. Such Pd-Ag direct exchange [Table 1(a)] is the predominant mechanism by which $\mathrm{Pd}$ atoms are incorporated into the Ag surface in the earlier stages. First, a Ag atom migrates out of the surface layer into a kink or a corner site present at the island edge. As seen by a modest energy barrier of $0.3 \mathrm{eV}$, such edge vacancies facilitate the otherwise constrained exchange process. The Ag pop-out creates a vacancy in the surface layer, which is quickly filled by a neighboring $\mathrm{Pd}$ atom. This $\mathrm{Pd}$ insertion has an energy barrier of only $0.05 \mathrm{eV}$, due to a large thermodynamic driving force (1.3 eV stabilization).

Next, some of the Ag atoms, now occupying the edge sites, become mobile and climb on top of the $\mathrm{Pd}$ deposit [Fig. 3(b)]. These Ag atoms are mobilized around kinks and corners, allowing the island edges to be rather fluxional. Adatom diffusion on close-packed surfaces has been investigated extensively in the computational literature. ${ }^{38}$ For example, our previous work has shown facile edge and corner diffusion of both Ag and Pd adatom on $\mathrm{Ag}$, with energy barriers below $0.4 \mathrm{eV}$ in all cases. ${ }^{21}$ For the simplest available benchmark of adatom self-diffusion on $\mathrm{Ag}(111)$, our energy barrier of $51 \mathrm{meV}$ is in good agreement with the experimental values reported in the range of 50-100 meV (see Lü et al. ${ }^{39}$ and the references therein).

Step ascent and descent are well-established in the context of island growth. ${ }^{40}$ There is a coordination loss and activation energy $\left(E_{\mathrm{a}}\right)$ associated with step descent. As such, the island growth mode is determined by the Ehrlich-Schwoebel barrier $\left(E_{\mathrm{ES}}\right),{ }^{41,42}$ defined as the additional activation energy required for step descent, relative to the terrace diffusion barrier $\left(E_{\mathrm{d}}\right)$ :

$$
E_{\mathrm{ES}}=E_{\mathrm{a}}-E_{\mathrm{d}}
$$

There are two main mechanisms of step ascent and descent: simple hopping over the edge [Table 1(c)], or exchange involving an insertion into the edge and a concurrent displacement of an edge atom [Table $1(\mathrm{~d})]$. Consistent with our previous work, ${ }^{21}$ we find that descent is more facile at threefold-terminated steps (B-step) than at fourfold-terminated steps (A-step), 
and that exchange is more facile than hopping. For the simplest case of Ag adatom selfdiffusion, our B-step Schwoebel barrier is $120 \mathrm{meV}$, in good agreement with the experimental value of $120 \pm 20 \mathrm{meV} .{ }^{43}$ To the best of our knowledge, no experimental benchmark exists for the diffusion of $\mathrm{Pd}$ adatom on $\mathrm{Ag}$.

In our simulation, exchange ascent and descent, followed by their hopping counterparts, comprise the majority of the observed restructuring events (Fig. S6). Interestingly, Ag atoms ascend primarily via hopping in the earlier stages, whereas exchange ascent occurs only when a sufficient number of Ag atoms are already present on the top to participate in the exchange. It is important to note that descent is easier than ascent in general, due to the larger coordination present at the lower edge than at the upper edge. However, an ascending $\mathrm{Ag}$ atom is capable of being stabilized by $\mathrm{Ag}$ atom(s) that may already be present on the top. Such extra coordination can lower the energy barrier by up to $0.3 \mathrm{eV}$ (Tables $\mathrm{S} 4$ and S5).

Surprisingly, the top Ag atoms pull up the underlying Pd atoms, ultimately forming a PdAg alloy layer in the second level [Fig. 3(c)]. This Pd ascent occurs primarily through a series of exchange involving the Ag atoms over a relatively short period of $0.2 \mu \mathrm{s}$, with energy barriers below $0.5 \mathrm{eV}$ [Table $1(\mathrm{~d})]$. The process is accompanied by a distinct relaxation of the deposit layer upon becoming coordinated by this second layer (Sec. 7, SI). Mechanistically, Pd hopping ascent occurs much less frequently, in contrast to Ag hopping ascent. In general, $\mathrm{Pd}$ atom experiences a larger energetic cost than Ag atom undergoing a given restructuring event, unless it is driven by a significantly large thermodynamic stabilization (e.g. Pd insertion into the surface). ${ }^{21}$

In the second half of the simulation, more Ag atoms migrate out of the surface to further cover the bilayer structure around its edges, resulting in a vacancy pit [Fig. 3(d)]. In all cases, Ag mass balance is conserved, and the number of Ag atoms that migrate out of the surface is roughly proportional to the number of $\mathrm{Pd}$ atoms deposited, e.g. $70 \mathrm{Ag}$ atoms migrate out of the surface in the case of $\mathrm{Pd}_{91}$ island (Fig. 3). As such, larger Pd islands 
lead to a larger amount of $\mathrm{Ag}$ transported out of the surface, creating a larger vacancy pit. Because our $\mathrm{Pd}_{91}$ island model is much smaller ( $\sim 3 \mathrm{~nm}$ wide) than the ones present in the experiment ( $\sim 50 \mathrm{~nm}$ wide), the resulting vacancy pit is also much smaller and localized in our simulation.

Our previous work found that Ag pop-out is very unfavorable on clean terraces, with energy barriers over $1.0 \mathrm{eV}$ in all scenarios. ${ }^{21}$ Surprisingly, a single-atom Ag pop-out next to an island edge has a relatively modest energy barrier of $0.6 \mathrm{eV}$ [Table 1(b)], which is attributed to the stabilization of the transition state by the edge atoms. The resulting vacancy pits create double steps through which facile Ag migration occurs, as proposed in previous experiments of rapid vacancy island decay. ${ }^{44,45}$ Remarkably, the thermodynamic driving force underlying this $\mathrm{Ag}$ transport becomes even more pronounced at higher $\mathrm{Pd}$ coverages (Sec. 6.3, SI). For example, at $0.75 \mathrm{ML}$ coverage, the surface area of the exposed $\mathrm{Ag}$ is less than that of the deposited Pd. Consequently, additional Ag migration begins to occur out of the subsurface layers, resulting in multiple-height steps and significant surface coarsening (Fig. S18).

At a relatively moderate temperature, the system has evolved into a completely different structure from the as-deposited Pd monolayer in a period of microseconds. At $500 \mathrm{~K}$, the system reaches an apparent quasi-equilibrium after $2 \mu \mathrm{s}$ in this metastable structure, as opposed to the thermodynamically favored state with Pd dispersed in the bulk. In particular, the exposed Pd clusters in the second layer are now much smaller and interspersed by $\mathrm{Ag}$ atoms. The observed structure is consistent with low-temperature CO probe experiments comprised of XPS, high resolution electron energy loss spectroscopy (HREELS), low energy ion scattering spectroscopy (LEISS), and temperature programmed desorption (TPD) - that collectively demonstrate significant reduction in CO binding after annealing at $450 \mathrm{~K}$ (Fig. 4).

The presence of surface $\mathrm{Pd}$ can be determined by probing the ability of $\mathrm{CO}$ to bind to the surface. CO adsorption occurs up to $100 \mathrm{~K}$ and $450 \mathrm{~K}$ on $\operatorname{Ag}(111)$ and $\operatorname{Pd}(111)$, respectively. ${ }^{46}$ As such, we expose $100 \mathrm{~L}\left(1\right.$ Langmuir $=1.0 \times 10^{-6}$ Torr s$\left.^{-1}\right)$ of $\mathrm{CO}$ to our 
(a)

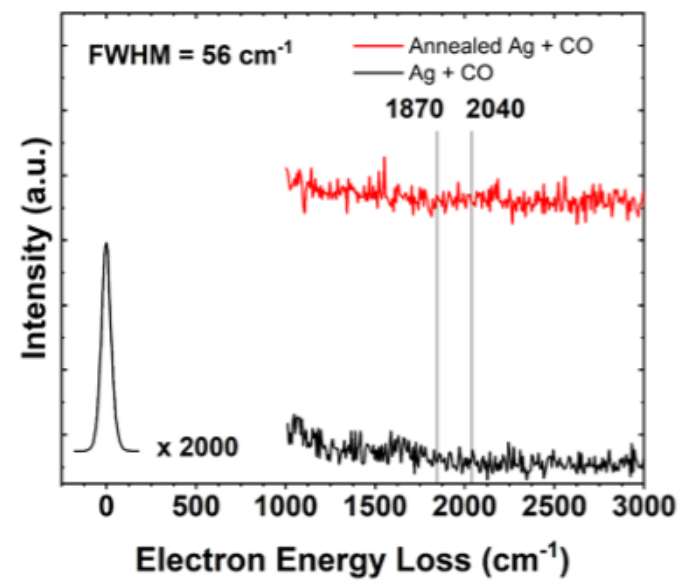

(c)

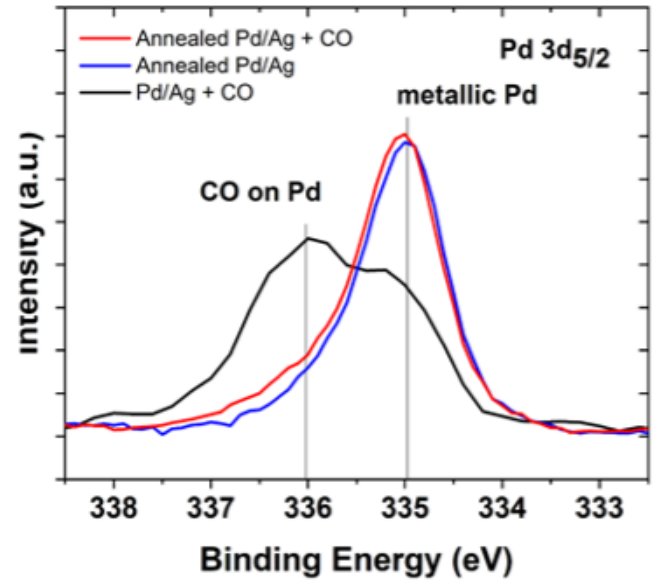

(b)

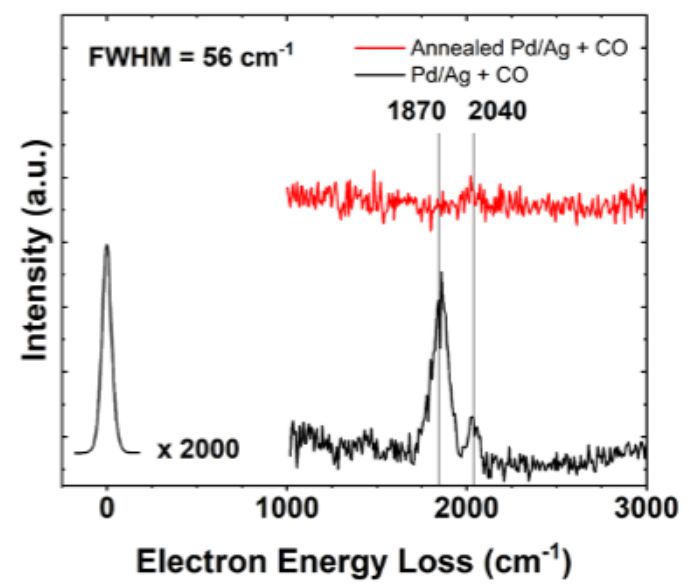

(d)

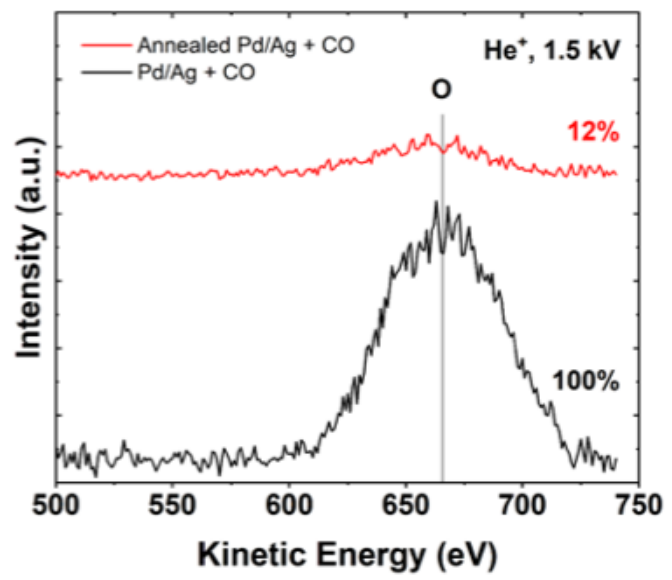

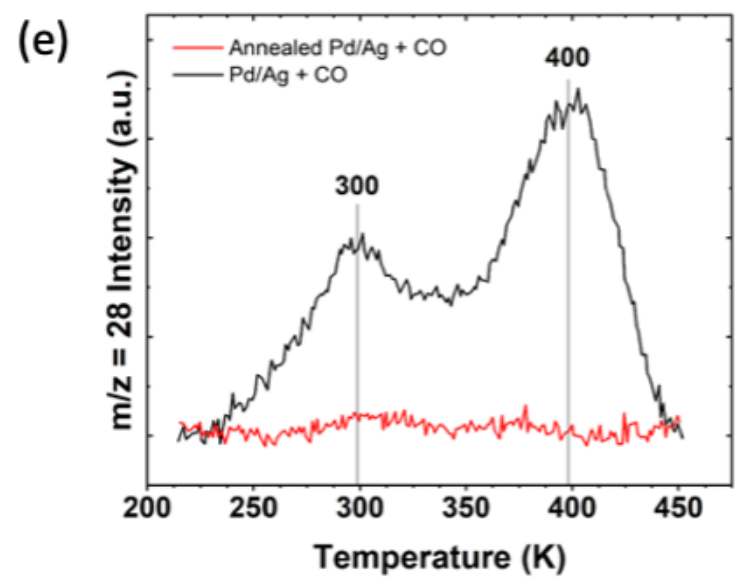

Figure 4: Adsorption of $\mathrm{CO}$ probes to detect the presence of surface $\mathrm{Pd}$ on $0.4 \mathrm{ML}$ $\mathrm{Pd} / \mathrm{Ag}(111)$. CO exposures are $100 \mathrm{~L}\left(1\right.$ Langmuir $=1.0 \times 10^{-6}$ Torr s$\left.^{-1}\right)$ at $100 \mathrm{~K}$. Surfaces are annealed by holding the sample at $450 \mathrm{~K}$ for $1 \mathrm{~min}$. (a) HREELS: $\mathrm{Ag}(111)$, as-prepared (black) and annealed (red), does not exhibit appreciable CO adsorption. (b) HREELS: $\mathrm{Pd} / \mathrm{Ag}(111)$, as-deposited (black) and annealed (red), exhibits $\mathrm{CO}$ adsorption on Pd bridge sites $\left(1870 \mathrm{~cm}^{-1}\right)$ and Pd top sites $\left(2040 \mathrm{~cm}^{-1}\right)$. (c) XPS: Clean Pd/Ag(111) annealed (blue); $\mathrm{CO}$ exposure to $\mathrm{Pd} / \mathrm{Ag}(111)$, as-deposited (black) and annealed (red). (d) LEISS: Quantitative CO coverage on $\mathrm{Pd} / \mathrm{Ag}(111)$, as-deposited (black) and annealed (red). (e) TPD: CO desorption from $\mathrm{Pd} / \mathrm{Ag}(111)$, as-deposited (black) and annealed (red). 
surfaces at $100 \mathrm{~K}$. The surfaces are prepared by depositing $0.4 \mathrm{ML}$ of $\mathrm{Pd}$ on $\mathrm{Ag}(111)$ at $300 \mathrm{~K}$, which is further annealed at $450 \mathrm{~K}$ for $1 \mathrm{~min}$. It is important to note that the $\mathrm{CO}$ exposure is performed at a low temperature of $100 \mathrm{~K}$ to limit thermal mobility of the system and thereby prevent any CO-induced restructuring of the surface, most notably reverse segregation of $\mathrm{Pd}$ to the surface. Previous studies have demonstrated such restructuring upon CO exposure at room temperature and above, for bimetallic systems including $\mathrm{Pd} / \mathrm{Ag},{ }^{10} \mathrm{Pt} / \mathrm{Au},{ }^{47} \mathrm{Ni} / \mathrm{Au},{ }^{48}$ and $\mathrm{Co} / \mathrm{Au} \cdot{ }^{49}$

$\mathrm{CO}$ does not adsorb appreciably on either as-prepared or annealed $\mathrm{Ag}(111)$ [Fig. 4(a)]. In contrast, $\mathrm{CO}$ adsorption occurs readily on as-deposited $\mathrm{Pd} / \mathrm{Ag}(111)$, as evidenced by a positive core-level shift in the $\mathrm{Pd} 3 d_{5 / 2}$ region, from $335.0 \mathrm{eV}$ of metallic $\mathrm{Pd}$ to $336.0 \mathrm{eV}$ upon CO exposure [Fig. 4(c); blue to black]. ${ }^{10,50}$ This observation is further supported by the elastic peak of oxygen arising from $\mathrm{CO}$ binding to Pd through its carbon and exposing its oxygen to the spectrometer [Fig. 4(d); black]. ${ }^{51}$ Specifically, CO adsorbs on Pd bridge sites and Pd top sites with vibrational frequencies of 1870 and $2040 \mathrm{~cm}^{-1}$, respectively, indicating the presence of extended Pd on the surface [Fig. 4(b); black]. ${ }^{46} \mathrm{CO}$ desorption at $300 \mathrm{~K}$ and $400 \mathrm{~K}$ further confirms the presence of CO adsorbed on Pd sites [Fig. 4(e); black]. ${ }^{46}$

However, $\mathrm{CO}$ adsorption is minimal on annealed $\mathrm{Pd} / \mathrm{Ag}(111)$, as evidenced by a very small positive core-level shift in the $\mathrm{Pd} 3 d_{5 / 2}$ region upon $\mathrm{CO}$ exposure, centered at $335.0 \mathrm{eV}$ of metallic Pd [Fig. 4(c); blue to red]. ${ }^{10,50}$ From quantification of the elastic peak of oxygen, only $12 \%$ of $\mathrm{CO}$ adsorbs on the annealed surface [Fig. 4(d); red], compared to the as-deposited surface, in very good agreement with our simulation predicting $\sim 7 \%$ of exposed Pd after annealing. Minimal CO desorption at $300 \mathrm{~K}$ and $400 \mathrm{~K}$ confirms a significant decrease in the surface concentration of Pd [Fig. 4(e); red]. In particular, only a trace amount of CO adsorption is detected on Pd top sites $\left(2040 \mathrm{~cm}^{-1}\right)$ [Fig. 4(b); red], suggesting that the annealed surface consists mostly of isolated Pd atoms and small Pd ensembles.

These experiments are also supported by DFT calculations showing a systematic reduction of $\mathrm{CO}$ adsorption energy upon decreasing the surface concentration of Pd (Table 2). 
Table 2: CO adsorption energy $\left(E_{\text {ads }}\right)$ on $\mathrm{Pd} / \mathrm{Ag}$ model surfaces, calculated with DFT using the M06L functional. ${ }^{52}$ All structures are fully optimized. The experimental benchmark for $1 / 4 \mathrm{ML} \mathrm{CO} / \mathrm{Pd}(111)$ is $-1.49 \mathrm{eV} .{ }^{53}$

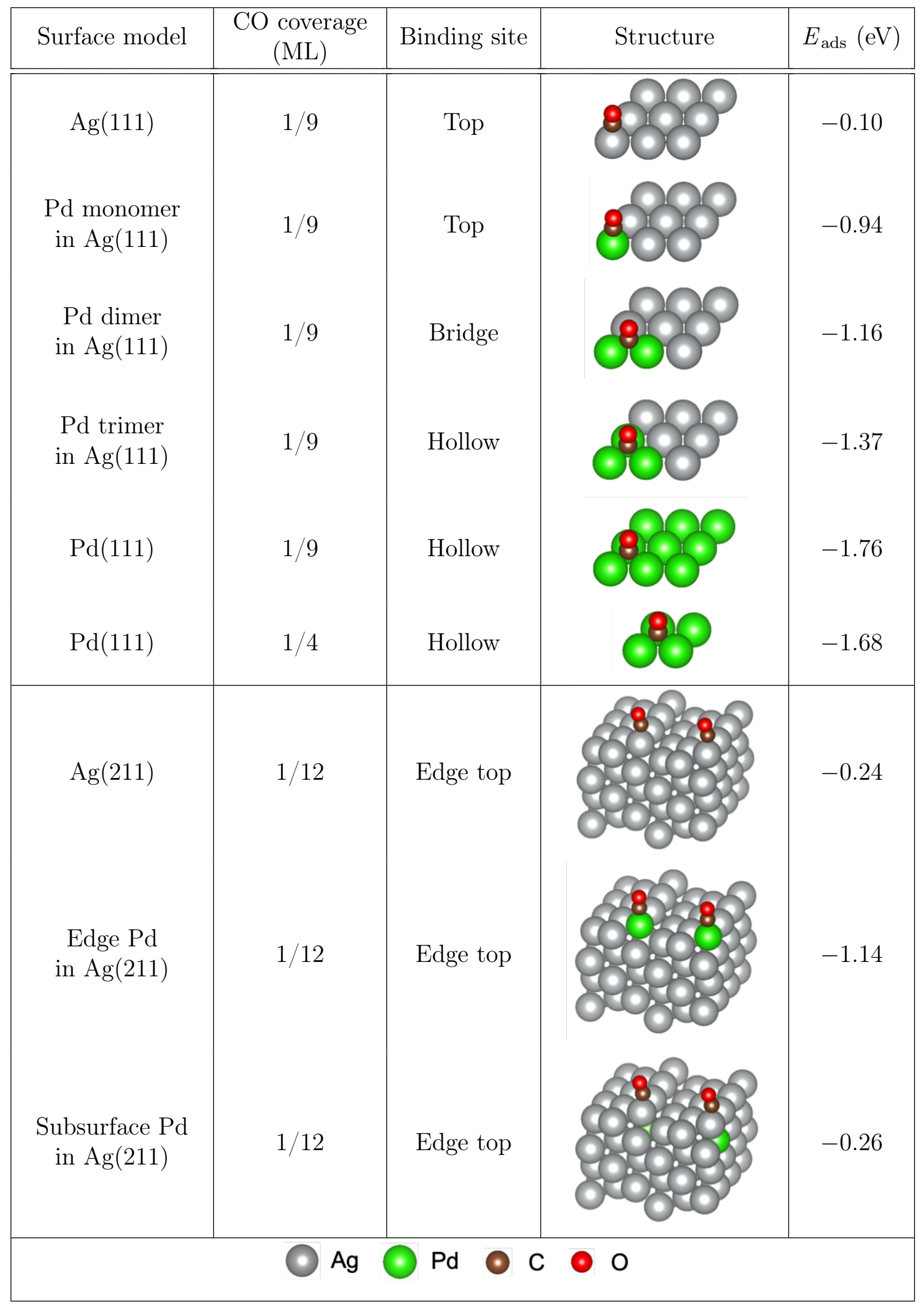


Based on carefully benchmarked results (Sec. 8, SI), the adsorption is dramatically weakened by the geometric effect, from $-1.76 \mathrm{eV}$ on an extended $\mathrm{Pd}(111)$ to $-0.94 \mathrm{eV}$ on an isolated $\mathrm{Pd}$ monomer in $\mathrm{Ag}(111)$, ultimately reaching $-0.10 \mathrm{eV}$ on a clean $\mathrm{Ag}(111)$. The same trend holds at the step edges, where the adsorption is slightly stronger by $\sim 0.2 \mathrm{eV}$ than on the terraces. Comparing an extended $\operatorname{Pd}(111)$ with a $\mathrm{Pd}$ trimer in $\operatorname{Ag}(111)$, the electronic effect also weakens the adsorption by $\sim 0.4 \mathrm{eV}$. Overall, these validations attest to the predictive power of our modeling method, complementary to experimental techniques with surface and chemical sensitivities.

At higher temperatures (Sec. 6.1, SI), the bilayer structure becomes further encapsulated by a third Ag capping layer, followed by layer-by-layer dissolution of Pd over a longer period of time (Fig. S12; see video here). The dissolution consists of multiple interlayer transfer events (Table S6), starting at the edges and moving toward the center of the island. A subsurface Ag atom first ascends into a pre-existing surface vacancy, after which a neighboring Ag-Pd pair descends into the resulting subsurface vacancy. As Pd dissolves layer-by-layer, it is no longer thermodynamically favorable for the $\mathrm{Ag}$ atoms to remain in the capping layer. As such, they migrate back into the surface, resulting in a gradual decay of vacancy pits. In the end, all pits are refilled, and almost all $\mathrm{Pd}$ remains dissolved in the subsurface, with a small amount of Pd resurfacing transiently as isolated atoms. Such surface-subsurface equilibrium is in direct agreement with the thermodynamic model of the quasi-chemical approximation. ${ }^{33}$

The formation of metastable Ag-encapsulated Pd islands is predominant at moderate temperatures and always precedes layer-by-layer dissolution of Pd. Such behavior has vital implications for catalysis. Our previous work showed that Pd resurfaces at moderate temperature and pressure of $\mathrm{CO}$ or $\mathrm{O}_{2} \cdot{ }^{10}$ This reverse segregation allows the catalytically active element to be present on the surface of an inert host metal under reaction conditions. Our results suggest that such activation of the catalyst would be enabled by metastable structures, where Pd remains largely accessible to the reactants in the form of Ag-encapsulated 
Pd rather than bulk-dissolved Pd. Evolution of these metastable structures would play a central mediating role in engineering of catalytic activity through various pretreatment strategies. ${ }^{54-57}$

\section{Conclusions}

For the first time, a detailed atomistic picture of the long-timescale restructuring of $\mathrm{Pd}$ on $\mathrm{Ag}$ is revealed, by combining microscopy, spectroscopy, and novel simulation methods. Because of its higher surface energy, Pd prefers to dissolve into the Ag host, ${ }^{10}$ but the underlying mechanism and timescale have remained speculative at best. We directly uncover both the mechanism and the timescale of the restructuring by developing and performing accelerated machine-learning molecular dynamics, in combination with surface science experiments. Using our newly developed automated analysis method, previously unidentified restructuring events have been discovered and characterized in an unbiased fashion, including Pd-Ag place exchange and Ag pop-out, as well as step ascent and descent. Remarkably, a significant amount of Ag migrates out of the surface to cover and alloy into the $\mathrm{Pd}$ islands, resulting in extensive vacancy pits within a period of microseconds. The microscopic insights established in this study are broadly applicable to other bimetallic systems comprised of soft metals, where surface segregation is primarily driven by the difference in the surface energy of the individual components.

Our automated modeling strategy within machine-learning molecular dynamics, in combination with surface science experiments, has allowed complex surface restructuring dynamics to be mapped out at atomic resolution, a task that would otherwise be intractable with a brute-force static approach. In this paper, the mechanisms for the reconstruction of a pristine $\mathrm{Pd} / \mathrm{Ag}(111)$ is demonstrated as a first step toward understanding the elementary events that lead to surface rearrangement. Investigation of more complex restructuring phenomena involving adsorbates is underway. In particular, the relatively low energy barriers of the 
discovered restructuring events suggest the possibility of catalytic processes proceeding on mobile surfaces, as seen in recent computational studies of nanocluster catalysis. ${ }^{58-60}$ The reactivity and the state of the surface may evolve together within a network of competition between thermodynamics and kinetics.

\section{Experimental Section}

Automated characterization of surface restructuring events: A fully automated workflow for surface restructuring event characterization has been developed (Fig. 1), building upon our previous framework. ${ }^{21}$ All of our interactive Jupyter Notebook files are publicly available. ${ }^{61}$ Density functional theory (DFT) data is used to train a Gaussian process (GP) machine-learning force field, ${ }^{23}$ which allows us to perform large-scale and long-timescale molecular dynamics (MD) at first-principles accuracy (Sec. 1, SI). From preliminary visual inspections, we find that most restructuring events occur over 1-3 ps, with atoms transitioning between well-defined face-centered cubic (FCC) lattice sites. To better follow the events, we first "clamp" the raw MD trajectory: All atoms are assigned onto discrete FCC lattice sites, frame by frame ${ }^{24-26}$ (Sec. 3, SI). The resulting clamped trajectory is automatically scanned to detect restructuring events. A frame is flagged as an event point if the $z$-coordinate (normal to the surface) of any atom changes irreversibly over 1-2 ps. The flagged frames within 2 ps of each other are clustered into distinct events. Then, the events are automatically sorted into seven main classes,${ }^{21}$ based on changes in the atomic layer and coordination number (Sec. 4, SI). Finally, pathways for representative events of each class are optimized using transition state modeling ${ }^{27-29}$ (Sec. 2, SI).

Surface science experiments: The experimental measurements are performed with three different $\mathrm{Ag}(111)$ crystals mounted in three separate ultrahigh vacuum (UHV) chambers, described in detail elsewhere. ${ }^{10,62}$ Scanning tunneling measurements are performed in a UHV chamber using a beetle-type scanner (RHK UHV VT STM). ${ }^{10}$ X-ray photoelectron and ion 
scattering measurements are performed in a UHV chamber using an X-ray source (Perkin Elmer 04-548/32-095 with RBD 20-042) with (non-monochromatized) Mg K $\alpha$ radiation, a $1.5 \mathrm{eV} 4 \mathrm{He}^{+}$ion beam (SPECS IQE 12/38) with a Wien mass filter (SPECS WF-IQE), and a hemispherical analyzer (SPECS Phoibos 100 equipped with a 5-channel electron multiplier). ${ }^{10}$ The high-resolution electron energy loss measurements are performed in a UHV chamber using an electron energy loss spectrometer (LK 2000) with a primary energy of $4.5 \mathrm{eV}$ at $60^{\circ}$ specular geometry. ${ }^{62}$ The $\mathrm{Ag}(111)$ crystals are cleaned by repeated cycles of $\mathrm{Ar}^{+}$sputtering $(1-2 \mathrm{kV})$ and annealing $(750-800 \mathrm{~K})$ in UHV. The PdAg surface alloys are formed by depositing $\mathrm{Pd}$ (99.99\%) at room temperature on clean $\mathrm{Ag}(111)$ using an electronbeam evaporator. CO adsorption experiments use a mixture of $20 \% \mathrm{CO}$ (99.9\% purity) in Ar.

\section{Additional information}

Supplementary information is available in the online version of the paper.

\section{Acknowledgment}

This work was supported by the Integrated Mesoscale Architectures for Sustainable Catalysis (IMASC), an Energy Frontier Research Center funded by the US Department of Energy (DOE), Office of Science, Office of Basic Energy Sciences under Award No. DE-SC0012573. J.V. was supported by Bosch Research. Y.X. was supported by the US Department of Energy (DOE), Office of Science, Office of Basic Energy Sciences under Award No. DE-SC0020128. This research used resources of the Advanced Light Source, a DOE Office of Science User Facility supported under Contract No. DE-AC02-05CH11231. J.S.L., J.V., Y.X., L.S., A.M., and J.F. used the Odyssey cluster, FAS Division of Science, Research Computing Group at Harvard University. J.S.L., L.S., and K.D. used the National Energy Research Scientific Computing Center (NERSC), a DOE Office of Science User Facility supported under 
Contract No. DE-AC02-05CH11231, through allocation m3275. K.D. used the Extreme Science and Engineering Discovery Environment (XSEDE), ${ }^{63}$ supported by National Science Foundation grant number ACI-1548562, through allocation che170060. We acknowledge enlightening discussions with M. Stamatakis, E. Kaxiras, S. B. Torrisi, W. Chen, and R. Réocreux.

\section{Competing financial interests}

The authors declare no competing financial interests.

\section{References}

(1) Dowben, P.; Miller, A. Surface Segregation Phenomena; Taylor \& Francis, 1990.

(2) Rodriguez, J. A. Physical and chemical properties of bimetallic surfaces. Surf. Sci. Rep. 1996, 24, 225-287.

(3) Zou, L.; Yang, C.; Lei, Y.; Zakharov, D.; Wiezorek, J. M. K.; Su, D.; Yin, Q.; Li, J.; Liu, Z.; Stach, E. A.; Yang, J. C.; Qi, L.; Wang, G.; Zhou, G. Dislocation nucleation facilitated by atomic segregation. Nat. Mater. 2018, 17, 56+, DOI: 10.1038/NMAT5034.

(4) Giannakakis, G.; Flytzani-Stephanopoulos, M.; Sykes, E. C. H. Single-Atom Alloys as a Reductionist Approach to the Rational Design of Heterogeneous Catalysts. Acc. Chem. Res. 2019, 52, 237-247, DOI: 10.1021/acs.accounts.8b00490.

(5) Aich, P.; Wei, H.; Basan, B.; Kropf, A. J.; Schweitzer, N. M.; Marshall, C. L.; Miller, J. T.; Meyer, R. Single-Atom Alloy Pd-Ag Catalyst for Selective Hydrogenation of Acrolein. J. Phys. Chem. C 2015, 119, 18140-18148, DOI: 10.1021/acs . jpcc. 5b01357. 
(6) Tao, F.; Grass, M. E.; Zhang, Y.; Butcher, D. R.; Renzas, J. R.; Liu, Z.; Chung, J. Y.; Mun, B. S.; Salmeron, M.; Somorjai, G. A. Reaction-Driven Restructuring of Rh-Pd and Pt-Pd Core-Shell Nanoparticles. Science 2008, 322, 932-934, DOI: $10.1126 /$ science. 1164170 .

(7) Tao, F.; Zhang, S.; Luan, N.; Zhang, X. Action of bimetallic nanocatalysts under reaction conditions and during catalysis: evolution of chemistry from high vacuum conditions to reaction conditions. Chem. Soc. Rev. 2012, 41, 7980-7993, DOI: $10.1039 / \mathrm{c} 2 \operatorname{cs} 35185 \mathrm{~d}$.

(8) Zafeiratos, S.; Piccinin, S.; Teschner, D. Alloys in catalysis: phase separation and surface segregation phenomena in response to the reactive environment. Catal. Sci. Technol. 2012, 2, 1787-1801, DOI: 10.1039/c2cy00487a.

(9) Vignola, E.; Steinmann, S. N.; Le Mapihan, K.; Vandegehuchte, B. D.; Curulla, D.; Sautet, P. Acetylene Adsorption on Pd-Ag Alloys: Evidence for Limited Island Formation and Strong Reverse Segregation from Monte Carlo Simulations. J. Phys. Chem. C 2018, 122, 15456-15463, DOI: 10.1021/acs.jpcc.8b04108.

(10) van Spronsen, M. A.; Daunmu, K.; O'Connor, C. R.; Egle, T.; Kersell, H.; OliverMeseguer, J.; Salmeron, M. B.; Madix, R. J.; Sautet, P.; Friend, C. M. Dynamics of Surface Alloys: Rearrangement of $\mathrm{Pd} / \mathrm{Ag}(111)$ Induced by CO and O-2. J. Phys. Chem. C 2019, 123, 8312-8323, DOI: 10.1021/acs.jpcc.8b08849.

(11) Murillo, L. E.; Goda, A. M.; Chen, J. G. Selective hydrogenation of the CO bond in acrolein through the architecture of bimetallic surface structures. J. Am. Chem. Soc. 2007, 129, 7101-7105, DOI: 10.1021/ja070264m.

(12) O'Connor, C. R.; van Spronsen, M. A.; Egle, T.; Xu, F.; Kersell, H. R.; OliverMeseguer, J.; Karatok, M.; Salmeron, M.; Madix, R. J.; Friend, C. M. Hydrogen migra- 
tion at restructuring palladiumâĂŞsilver oxide boundaries dramatically enhances reduction rate of silver oxide. Nat. Commun. 2020, 11, DOI: 10.1038/s41467-020-15536-x.

(13) Zugic, B.; Wang, L.; Heine, C.; Zakharov, D. N.; Lechner, B. A. J.; Stach, E. A.; Biener, J.; Salmeron, M.; Madix, R. J.; Friend, C. M. Dynamic restructuring drives catalytic activity on nanoporous gold-silver alloy catalysts. Nat. Mater. 2017, 16, 558+, DOI: $10.1038 /$ NMAT4824.

(14) Personick, M. L.; Montemore, M. M.; Kaxiras, E.; Madix, R. J.; Biener, J.; Friend, C. M. Catalyst design for enhanced sustainability through fundamental surface chemistry. Philos. Trans. Royal Soc. A 2016, 374, DOI: 10.1098/rsta.2015.0077.

(15) Kim, H. Y.; Henkelman, G. CO Adsorption-Driven Surface Segregation of Pd on Au/Pd Bimetallic Surfaces: Role of Defects and Effect on CO Oxidation. ACS Catal. 2013, 3, 2541-2546, DOI: $10.1021 / \operatorname{cs} 4006259$.

(16) Cheng, F.; He, X.; Chen, Z.-X.; Huang, Y.-G. Kinetic Monte Carlo simulation of surface segregation in Pd-Cu alloys. J. Alloys and Compd. 2015, 648, 1090-1096, DOI: $10.1016 / \mathrm{j} \cdot \mathrm{jallcom} .2015 .05 .286$.

(17) An, H.; Ha, H.; Yoo, M.; Kim, H. Y. Understanding the atomic-level process of COadsorption-driven surface segregation of $\mathrm{Pd}$ in $(\mathrm{AuPd})(147)$ bimetallic nanoparticles. Nanoscale 2017, 9, 12077-12086, DOI: 10.1039/c7nr04435f.

(18) Xu, C.-Q.; Lee, M.-S.; Wang, Y.-G.; Cantu, D. C.; Li, J.; Glezakou, V.-A.; Rousseau, R. Structural Rearrangement of Au-Pd Nanoparticles under Reaction Conditions: An ab Initio Molecular Dynamics Study. ACS Nano 2017, 11, 1649-1658, DOI: $10.1021 /$ acsnano.6b07409.

(19) Wexler, R. B.; Qiu, T.; Rappe, A. M. Automatic Prediction of Surface Phase Diagrams Using Ab lnitio Grand Canonical Monte Carlo. J. Phys. Chem. C 2019, 123, 23212328, DOI: $10.1021 /$ acs . jpcc.8b11093. 
(20) Yang, Y. and Shen, X. and Han, Y.-F., Diffusion mechanisms of metal atoms in PdAu bimetallic catalyst under CO atmosphere based on ab initio molecular dynamics. Appl. Surf. Sci. 2019, 483, 991-1005, DOI: 10.1016/j.apsusc.2019.04.036.

(21) Lim, J. S.; Molinari, N.; Duanmu, K.; Sautet, P.; Kozinsky, B. Automated Detection and Characterization of Surface Restructuring Events in Bimetallic Catalysts. J. Phys. Chem. C 2019, 123, 16332-16344, DOI: 10.1021/acs.jpcc.9b04863.

(22) Vignola, E.; Steinmann, S. N.; Al Farra, A.; Vandegehuchte, B. D.; Curulla, D.; Sautet, P. Evaluating the Risk of C-C Bond Formation during Selective Hydrogenation of Acetylene on Palladium. ACS Catal. 2018, 8, 1662-1671, DOI: 10.1021/acscatal.7b03752.

(23) Vandermause, J.; Torrisi, S. B.; Batzner, S.; Xie, Y.; Sun, L.; Kolpak, A. M.; Kozinsky, B. On-the-fly active learning of interpretable Bayesian force fields for atomistic rare events. NPJ Comput. Mater. 2020, 6, DOI: 10.1038/s41524-020-0283-z.

(24) Kahle, L.; Musaelian, A.; Marzari, N.; Kozinsky, B. Unsupervised landmark analysis for jump detection in molecular dynamics simulations. Phys. Rev. Mater. 2019, 3, 055404, DOI: 10.1103/PhysRevMaterials .3.055404.

(25) Musaelian, A.; Kahle, L. "sitator". 2018, https://github.com/Linux-cpp-lisp/sitator.

(26) Musaelian, A. "surfator". 2019, https://github.com/mir-group/surfator.

(27) Henkelman, G.; Uberuaga, B. P.; Jonsson, H. A climbing image nudged elastic band method for finding saddle points and minimum energy paths. J. Chem. Phys. 2000, 113, 9901-9904, DOI: 10.1063/1.1329672.

(28) Henkelman, G.; Jonsson, H. Improved tangent estimate in the nudged elastic band method for finding minimum energy paths and saddle points. J. Chem. Phys. 2000, 113, 9978-9985, DOI: 10.1063/1.1323224. 
(29) Henkelman, G.; Jonsson, H. A dimer method for finding saddle points on high dimensional potential surfaces using only first derivatives. J. Chem. Phys. 1999, 111, 7010-7022, DOI: $10.1063 / 1.480097$.

(30) Shiihara, Y.; Kohyama, M. Contribution of d electrons to surface stresses and their changes by layer relaxation for a series of $4 \mathrm{~d}$ transition metals. Surf. Sci. 2016, 644, 122-128, DOI: $10.1016 /$ j.susc. 2015.08.009.

(31) Bach, C. E.; Giesen, M.; Ibach, H.; Einstein, T. L. Stress relief in reconstruction. Phys. Rev. Lett. 1997, 78, 4225-4228, DOI: 10.1103/PhysRevLett.78.4225.

(32) Treglia, G.; Legrand, B.; Ducastelle, F.; Saul, A.; Gallis, C.; Meunier, I.; Mottet, C.; Senhaji, A. Alloy surfaces: segregation, reconstruction and phase transitions. Comput. Mater. Sci. 1999, 15, 196-235, DOI: 10.1016/S0927-0256(99)00004-X.

(33) Creemers, C.; Deurinck, P. Platinum segregation to the (111) surface of ordered Pt80Fe20: LEIS results and model simulations. Surf. Interface Anal. 1997, 25, 177190, DOI: 10.1002/(SICI) 1096-9918(199703) $25: 3<177:$ :AID-SIA219>3 . 0 . CO ; 2-T, QSA-9 Conference, Guildford, England, Jul. 15-19, 1996.

(34) Hirschl, R.; Delbecq, F.; Sautet, P.; Hafner, J. Adsorption of unsaturated aldehydes on the (111) surface of a Pt-Fe alloy catalyst from first principles. J. Catal. 2003, 217, 354-366, DOI: 10.1016/S0021-9517(03)00057-5.

(35) Divi, S.; Chatterjee, A. Generalized nano-thermodynamic model for capturing sizedependent surface segregation in multi-metal alloy nanoparticles. $R S C A d v$. 2018, 8 , 10409-10424, DOI: $10.1039 /$ c8ra00945g.

(36) Vignola, E.; Steinmann, S. N.; Vandegehuchte, B. D.; Curulla, D.; Sautet, P. C2H2Induced Surface Restructuring of Pd-Ag Catalysts: Insights from Theoretical Modeling. J. Phys. Chem. C 2016, 120, 26320-26327, DOI: 10.1021/acs.jpcc.6b08524. 
(37) Roussel, J. M.; Saul, A.; Treglia, G.; Legrand, B. Layer-by-layer versus surfactant dissolution modes in heteroepitaxy. Phys. Rev. B 1999, 60, 13890-13901, DOI: 10.1103/PhysRevB.60.13890.

(38) Kim, S. Y.; Lee, I.-H.; Jun, S. Transition-pathway models of atomic diffusion on fcc metal surfaces. II. Stepped surfaces. Phys. Rev. B 2007, 76, DOI: 10.1103/PhysRevB.76.245408.

(39) Lu, B.; Almyras, G. A.; Gervilla, V.; Greene, J. E.; Sarakinos, K. Formation and morphological evolution of self-similar 3D nanostructures on weakly interacting substrates. Phys. Rev. Mater. 2018, 2, DOI: 10.1103/PhysRevMaterials. 2.063401.

(40) Li, Y. G.; DePristo, A. E. Predicted growth mode for metal homoepitaxy on the fcc(111) surface. Surf. Sci. 1996, 351, 189-199, DOI: 10.1016/0039-6028(96)80063-X.

(41) Ehrlich, G.; Hudda, F. G. Atomic view of surface self-diffusion - tungsten on tungsten. J. Chem. Phys. 1966, 44, 1039-\&, DOI: 10.1063/1.1726787.

(42) Schwoebel, R. L.; Shipsey, E. J. Step motion on crystal surfaces. J. Appl. Phys. 1966, 37, 3682+, DOI: 10.1063/1.1707904.

(43) Bromann, K.; Brune, H.; Roder, H.; Kern, K. Interlayer Mass Transport in Homoepitaxial and Heteroepitaxial Metal Growth. Phys. Rev. Lett. 1995, 75, 677-680, DOI: 10.1103/PhysRevLett.75.677.

(44) Giesen, M.; Ibach, H. On the mechanism of rapid mound decay. Surf. Sci. 2000, 464, L697-L702, DOI: 10.1016/S0039-6028(00)00693-2.

(45) Shen, M.; Jenks, C. J.; Evans, J. W.; Thiel, P. A. Rapid decay of vacancy islands at step edges on $\operatorname{Ag}(111)$ : step orientation dependence. J. Phys. Condens. Matter 2010, 22, DOI: $10.1088 / 0953-8984 / 22 / 21 / 215002$. 
(46) Ma, Y.; Diemant, T.; Bansmann, J.; Behm, R. J. The interaction of CO with $\mathrm{PdAg} / \mathrm{Pd}(111)$ surface alloys-A case study of ensemble effects on a bimetallic surface. Phys. Chem. Chem. Phys. 2011, 13, 10741-10754, DOI: 10.1039/c1cp00009h.

(47) Tenney, S. A.; Ratliff, J. S.; Roberts, C. C.; He, W.; Ammal, S. C.; Heyden, A.; Chen, D. A. Adsorbate-Induced Changes in the Surface Composition of Bimetallic Clusters: Pt-Au on TiO2(110). J. Phys. Chem. C 2010, 114, 21652-21663, DOI: 10.1021/jp108939h.

(48) Tenney, S. A.; He, W.; Roberts, C. C.; Ratliff, J. S.; Shah, S. I.; Shafai, G. S.; Turkowski, V.; Rahman, T. S.; Chen, D. A. CO-Induced Diffusion of Ni Atoms to the Surface of Ni-Au Clusters on TiO2(110). J. Phys. Chem. C 2011, 115, 11112-11123, DOI: $10.1021 / j p 2014258$.

(49) Galhenage, R. P.; Ammal, S. C.; Yan, H.; Duke, A. S.; Tenney, S. A.; Heyden, A.; Chen, D. A. Nucleation, Growth, and Adsorbate-Induced Changes in Composition for Co-Au Bimetallic Clusters on TiO2. J. Phys. Chem. C 2012, 116, 24616-24629, DOI: 10.1021/jp307888p.

(50) Surnev, S.; Sock, M.; Ramsey, M. G.; Netzer, F. P.; Wiklund, M.; Borg, M.; Andersen, J. N. CO adsorption on $\mathrm{Pd}(111)$ : a high-resolution core level photoemission and electron energy loss spectroscopy study. Surf. Sci. 2000, 470, 171-185, DOI: $10.1016 / \mathrm{S} 0039-6028(00) 00853-0$.

(51) Shen, Y. G.; O'Connor, D. J.; Wandelt, K.; MacDonald, R. J. CO adsorption on Cu3Pt(111): a LEIS study. Surf. Sci. 1995, 331, 746-752, DOI: 10.1016/0039-6028(95)00149-2, 14th European Conference on Surface Science (ECOSS-14), Leipzig, Germany, Sep 19-23, 1994.

(52) Zhao, Y.; Truhlar, D. G. A new local density functional for main-group thermochem- 
istry, transition metal bonding, thermochemical kinetics, and noncovalent interactions. J. Chem. Phys. 2006, 125, DOI: 10.1063/1.2370993.

(53) Wellendorff, J.; Silbaugh, T. L.; Garcia-Pintos, D.; Norskov, J. K.; Bligaard, T.; Studt, F.; Campbell, C. T. A benchmark database for adsorption bond energies to transition metal surfaces and comparison to selected DFT functionals. Surf. Sci. 2015, 640,36-44, DOI: 10.1016/j.susc.2015.03.023.

(54) Foettinger, K.; van Bokhoven, J. A.; Nachtegaal, M.; Rupprechter, G. Dynamic Structure of a Working Methanol Steam Reforming Catalyst: In Situ QuickEXAFS on Pd/ZnO Nanoparticles. J. Phys. Chem. Lett. 2011, 2, 428-433, DOI: $10.1021 / j z 101751 \mathrm{~s}$.

(55) Pan, Y.-T.; Yan, L.; Shao, Y.-T.; Zuo, J.-M.; Yang, H. Regioselective Atomic Rearrangement of Ag-Pt Octahedral Catalysts by Chemical Vapor-Assisted Treatment. Nano Lett. 2016, 16, 7988-7992, DOI: 10.1021/acs.nanolett.6b04411.

(56) Bedford, N. M.; Showalter, A. R.; Woehl, T. J.; Hughes, Z. E.; Lee, S.; Reinhart, B.; Ertem, S. P.; Coughlin, E. B.; Ren, Y.; Walsh, T. R.; Bunker, B. A. Peptide-Directed PdAu Nanoscale Surface Segregation: Toward Controlled Bimetallic Architecture for Catalytic Materials. ACS Nano 2016, 10, 8645-8659, DOI: 10.1021/acsnano.6b03963.

(57) Zugic, B. et al. Evolution of steady-state material properties during catalysis: Oxidative coupling of methanol over nanoporous Ag0.03Au0.97. J. Catal. 2019, 380, 366-374, DOI: $10.1016 / j \cdot j$ cat . 2019.08 .041 .

(58) Zhao, W.; Chizallet, C.; Sautet, P.; Raybaud, P. Dehydrogenation mechanisms of methyl-cyclohexane on ÂÔÂş-Al2O3 supported Pt13: Impact of cluster ductility. J. Catal. 2019, 370, 118 - 129, DOI: https://doi.org/10.1016/j.jcat.2018.12.004. 
(59) Sun, G.; Alexandrova, A. N.; Sautet, P. Structural Rearrangements of Subnanometer $\mathrm{Cu}$ Oxide Clusters Govern Catalytic Oxidation. ACS Catal. 2020, 10, 5309-5317, DOI: 10.1021/acscatal.0c00824.

(60) Guo, H.; Sautet, P.; Alexandrova, A. N. Reagent-Triggered Isomerization of Fluxional Cluster Catalyst via Dynamic Coupling. J. Phys. Chem. Lett. 2020, 11, 3089-3094, DOI: $10.1021 /$ acs.jpclett.0c00548.

(61) Lim, J. S. "Automated surface restructuring event characterization". 2019, https://github.com/mir-group/surface-restructuring.

(62) Rodriguez-Reyes, J. C. F.; Friend, C. M.; Madix, R. J. Origin of the selectivity in the gold-mediated oxidation of benzyl alcohol. Surf. Sci. 2012, 606, 1129-1134, DOI: $10.1016 / j$. susc. 2012.03 .013 .

(63) Towns, J.; Cockerill, T.; Dahan, M.; Foster, I.; Gaither, K.; Grimshaw, A.; Hazlewood, V.; Lathrop, S.; Lifka, D.; Peterson, G. D.; Roskies, R.; Scott, J. R.; WilkinsDiehr, N. XSEDE: Accelerating Scientific Discovery. Comput. Sci. Eng. 2014, 16, $62-$ 74, DOI: $10.1109 /$ MCSE. 2014.80. 